\title{
D esigualdades sociais e gestão em saúde: metodologia de seleção de áreas urbanas visando à diminuição das desigualdades socioespaciais em regiões metropolitanas
}

\author{
Social inequalities and administration in health: \\ methodology of selection of urban areas seeking \\ to the decrease of the partner-space inequalities \\ in metropolitan areas
}

1 Departamento de Ciências Sociais, ENSP, Fiocruz. Rua Leopoldo Bulhões 1480, sala 917 $M$ anguinhos, 21041-210, Rio de Janeiro RJ. najar@ensp.fiocruz.br
Abstract This article presents a methodology to study the Rio de Janei ro Greater M etropolitan Area, termed "Rio, $40^{\circ} \mathrm{C}$ ", using the census tract as the data aggregation unit, making access available to an adjuvant tool in the process of allocating health management resources with the objective of minimizing social in equalities expressed in urban space, especially metropolitan urban areas. From this perspective, all the M unicipal and State departments comprising the social area of a given government administration can take advantage of the methodology presented here. The techniques used are: exploratory data analysis, generation of basic indicators, principal components analysis, and cluster analysis. The level of detail achieved is a function of the scale of data aggregation in the census tract. The final product was developed and implemented in an electronic medium (CD-ROM) containing all the data bases, allowing data analysis according to the user's needs, with special emphasis on planning of social actions and programs and decision-making support. The final result will support considerations on the central position of metropolitan space in the analysis of quality-of-life profiles, analysis of public investments, and electoral analyses.

Key words Socio-spatial inequalities, AnaIytical methodology, M etropolitan area, Information systems, Quantitative methods in public health
Resumo 0 artigo apresenta metodologia, à qual se deu o nome de Rio 40 graus, para estudar a região metropolitana do Rio de Janeiro, utilizando como unidade de agregação da informação o setor censitário, di sponibilizando 0 acesso a uma ferramenta coadjuvante no processo de alocação de recursos e de gestão em saúde. 0 objeti vo é minimizar as desigualdades de natureza social, sendo de especial interesse para o espaço urbano-metropolitano. N essa perspectiva, todas as secretarias, municipais e estaduais, que integram a área social de um governo, podem tirar proveito de tal metodologia. As técnicas utilizadas são: análise exploratória de dados, geração de indicadores básicos, análise de componentes principais e cluster. 0 nível de detalhe alcançado é função da escala de agregação dos dados do setor censitário. 0 produto final foi desenvolvido e implementado em mídia eletrônica (CD-ROM), contendo todas as bases de dados, permitindo a análise das mesmas segundo necessidades do usuário, com ênfase especial no planejamento de ações e programas sociais e apoi o à decisão. 0 resultado final pode oferecer suporte a análises do espaço metropolitano, de perfis de qualidade de vida, de investimentos públicos, eleitorais, dentre outras.

Palavras-chave Desigual dades socioespaciais, M etodologia de análise, Região metropolitana, Sistemas de informações, M étodos quantitativos em saúde pública 


\section{Introdução}

0 artigo apresenta em largos traços metodologia desenvolvida em trabalhos anteriores que permite hierarquizar áreas da região metropolitana do Rio de Janeiro (setores censitários, bairros e regiões administrativas) segundo um índice de qualidade social construído a partir de variáveis censitárias. Essa mesma metodologia ensejou a criação de um software de visualização para a região metropolitana do Rio de Janeiro (N ajar et al., 2000).

0 artigo tem como escopo duas motivações principais. A primeira, contribuir para recuperar o nível analítico metropolitano na equação dos problemas de saúde nas grandes cidades brasileiras, secundarizado, pelo menos do ponto de vista político-administrativo, depois da Constituição de 1988, quando instituições com área de abrangência metropolitana, que, de alguma maneira constituíam um quarto nível de gestão, pelo menos do ponto de vista gerencial, foram-se esvaziando. A segunda, propiciar acesso a uma técnica de visualização da informação quantitativa, na discussão da economia da saúde e na diminuição das desigualdades de natureza socioespacial, não só desenvolvendo uma metodologia de identificação da vulnerabilidade social, como também oferecendo uma ferramenta para seu mapeamento e análise, endereçada especial mente aos gestores públicos envolvidos na tarefa de, por um lado, identificar padrões de distribuição de variáveis socioeconômicas, e de, por outro lado, indicar áreas vulneráveis do ponto de vista social. Assim, este trabalho alinha-se, sob o aspecto da tradição metodológica, aos importantes trabalhos de Vetter (1981) e Pinçon-Charlot et al. (1986).

U ma questão de natureza metodológica quanto ao emprego de variáveis com expressão espacial relaciona-se com a complexidade metropolitana, que levou boa parte das análises a tratar como similares espaços dessemel hantes sob o ponto de vista de sua composição e constituição social. Já no início da década de 1980, Vetter (1981) advertia a respeito, assinalando a existência não de um núcleo e de sua periferia mas sim de um sistema de núcleos e periferias. M arques (1993) pode comprovar a existência, já em 1980, de conteúdos sociais diversos em espaços considerados homogêneos pela literatura. A estruturação interna da região metropolitana do Rio de Janeiro era aparentemente não concêntrica e não contígua entre espaços com conteúdos sociais similares, se assemel hando muito mais a um mosaico complexo do que a um gradiente decrescente de círculos concêntricos. Foi exatamente esse aspecto que se revelou também em outras análises, tais como $\mathrm{N}$ ajar (1997, 1998 e 2000): 0 aglomerado metropolitano comandado pela cidade do Rio de Janeiro se apresentou, não como uma cidade segregada, partida (Ventura, 1994), mas como um verdadeiro e sofisticado mosaico urbano (Timms, 1971), marcado tanto por dégradés quanto por dicotomias, desautorizando-nos a pensar o espaço urbano carioca como uma cidade dual, visto que ele se nos apresentava como uma cerzidura, um mosaico complexo, bem mais próximo ao que parece ter-se transformado a própria sociedade brasileira nas últimas décadas.

A análise apoiou-se em um sistema de informações geográficas da região metropolitana do Rio de Janeiro com o objetivo específico de estabelecer uma base socioeconômica com referência espacial para a identificação de grupos e segmentos do território, supostamente mais vulneráveis sob o ponto de vista social. Com essa finalidade utilizaram-se ferramentas de análise multivariada para a caracterização da estrutura socioespacial dessa mesma região propondo-se um modelo analítico que parte de uma premissa simples eamplamente aceita na sociologia, qual seja: de que a distribuição dos investimentos públicos guarda relação estreita com a estrutura social corporificada no espaço ou, segundo Vetter (1982), de que há uma “causação circular" (semelhante à causação reversa da epidemiologia) provocada por uma superposição entre renda real e renda monetária. Acrescentamos que essa renda real, no caso das políticas públicas de saúde, são benefícios líquidos que podem se traduzir em mudanças dos perfis epidemiológicos da população. D ada à melhoria general izada das condições de vida da população nas grandes cidades brasileiras, essas mudanças só podem ser detectadas, num sentido operacional que interessa grandemente à saúde pública, caso se trabalhe com níveis de agregação convenientes, por exemplo, setores censitários ou domicílios, que poderão ser posteriormente reagrupados através de técnicas especiais - análise de clusters, por exemplo - , oferecendo assim novas "visões" sobre os aglomerados urbanos, no caso, regiões metropolitanas.

Por essa razão, a construção da base partiu de um cruzamento de indicadores sociais, de forma a que se pudesse analisar a distribuição espacial das variáveis. Para gerar uma caracterização mais abrangente, a abordagem central do 
trabalho foi pautada na construção de uma classificação ascendente hierárquica a partir de uma análise de componentes principais, também utilizada por diversos trabalhos recentes, em especial Preteceillee Valladares (2000).

A abordagem proposta apresenta um índice, o IQS - Índice de Qualidade Social -, que permite a visualização da região metropolitana do Rio de Janeiro com nível de detal he de setor censitário. Isso significa o exame de áreas que agregam entre 300 a 400 domicílios. Em seguida, foi elaborada a tipologia das unidades espaciais - setores censitários, bairros, regiões administrativas e municípios -, segundo seus conteúdos socioeconômicos expressos através de variáveis agrupadas a partir dos seguintes blocos temáticos: demografia; renda; educação; infra-estrutura e serviços; e, condições da habitação/domicílio. A pós a utilização de análise de componentes principais e análise de cluster, 0 trabalho resultou no estabelecimento de sete grupos de unidades. 0 resultado final é um índice sintetizador que permite classificar as unidade espaciais utilizadas segundo uma escala de categorias que, respeitados os seus limites, pode ser usado como proxy da qualidade social do território em exame.

0 resultado da classificação acabou por revelar importantes transformações na forma como classicamente a literatura interpretou a distribuição da estrutura social no espaço metropolitano carioca (Abreu, 1987). Os modelos utilizados consideram uma distribuição concêntrica, contínua e contígua entre espaços de características sociais similares a partir do centro da metrópole (M arques, 1998). Os indicadores apontam para a existência de um tecido social muito mais complexo no espaço da metrópole, no qual áreas de características similares se encontram em locais não contíguos. E os eixos de transportes (e seus custos) claramente, e com freqüência, não são o único nem 0 mais importante fator na localização das atividades e grupos no espaço. Embora as causas do padrão observado não tenham sido discutidas, é razoável sustentar a hipótese de que fatores ligados à história da cidade e à inércia dos diversos momentos da estruturação do espaço, as estratégias, interesses e valores de cada grupo social (referentes a cada espaço) e à cultura urbana conformem o núcleo causal da estruturação do espaço metropolitano.

Ao investigar a diferenciação interna na região, os resultados da análise confirmaram a constatação de diversos trabalhos que indicam, por exemplo, que há bairros e favelas dentro de uma mesma favela (Preteceille \& V alladares, 2000; M arques, 1993; V etter, 1981 e M achado da Silva, 1967). Especialmente com relação às favelas cariocas, os resultados a que chegamos, assim como outros tantos trabalhos - Preteceille \& Valladares (2000), Valladares, 2001 - recomendam que se deveter cuidado ao se discutir a periferização das favelas em relação à metrópole.

0 grande potencial de crescimento e rendimento das análises sugere, e quase impõe, uma agenda de desenvolvimento pautada principalmente pela comparação de três anos censitários - 1991, 1996 e 2000 -, o que permitirá a comparação de uma década, a partir de um mesmo modelo e com uma mesma metodologia. A comparação 1991/1996 já foi objeto de estudo ( $N$ ajar et al., 2002).

\section{Discussão: modelos de análise socioespaciais}

Para uma apreciação crítica dos resultados aqui apresentados, deve-se fazer referência a uma discussão que tem longa tradição sociológica. $M$ arques (1998) sugere dividir a literatura relevante sobre a estruturação intra-urbana em grandes cidades em três grandes grupos. 0 primeiro se dedica a estudar o crescimento histórico das cidades, tentando descrever as diversas dinâmicas encontradas. Esses estudos são patrimônio da tradição arquitetônica/urbanística e recebem normalmente a designação de estudos sobre forma urbana.

Um segundo grupo aborda a estruturação interna das cidades com caráter propositivo e normativo. Trata-se de análises que, mesmo quando voltadas para o passado, pretendem projetar ou planejar o futuro das cidades. Dentro dessa linha, pode-se incluir toda a tradição urbanística e do planejamento urbano stricto sensu, ou seja, a produção de modelos, análises e instrumentos que, uma vez implementados, possibilitam alterar a estruturação urbana em um certo sentido predeterminado.

0 terceiro grupo refere se a tentativas de re produção, em modelos, do padrão de estruturação urbana percebido e mediado pelos sentidos e pelo pensamento. Esses modelos se apóiam na observação e no estudo de grande número de análises do primeiro grupo (históricos e de forma urbana) e se relacionam com os do segundo tipo (normativos e propositivos), mas constituem um campo próprio com interesses espe- 
cíficos, tanto analíticos quanto explicativos. Abordagens desse tipo são eminentemente teóricas e compostas por dois momentos. Em uma primeira fase, orientadas por hipóteses, produzem um modelo, e em um segundo momento, orientadas pelos dados, comparam o modelo às situações empíricas.

No conjunto de saberes dos estudos urbanos, nem todos os estudiosos produziram modelos de estruturação. Isso se deve às diferentes preocupações presentes, assim como a diferentes posturas com relação à atividade de pesquisa. Tradições de caráter cientificista tendem a procurar padrões e leis gerais, ao contrário de tradições empiristas. Tradições que não privilegiam a natureza autônoma do espaço urbano, por outro lado, tendem a rejeitar a construção de modelos. A combinação dessas duas características tem definido a existência de diversos modelos.

Segundo Park (1925) a cidade é um estado de espírito, é um produto da natureza, e particularmente da natureza humana. Essa natureza seria composta por características biológicas e culturais. Às primeiras estaria associada uma volição à competição, que alocaria os indivíduos no espaço segundo suas aptidões. À segunda estaria relacionada a necessidade de comunicação, a de interação social e da construção de uma ordem moral.

Os autores da Escola de Chicago realizaram um extenso trabalho empírico sobre cultura urbana, tentando determinar a especificidade do "urbanismo como modo de vida". Esses estudos focalizaram os diversos comportamentos na comunidade urbana, a vizinhança, a delinqüência, a mobilidade intra-urbana, a vida nos bairros fortemente segregados etnicamente, etc. Para Velho (1973), os estudos realizados pelos principais autores do movimento - Robert Park, Ernest Burguess, Rodrick M cKenzie e principalmente Louis Wirth - representaram as primeiras análises de sociologia urbana. Foi realizado também um esforço de generalização, tentando construir uma teoria científica do crescimento urbano e da estruturação espacial baseada nas cidades norte-americanas. A localização no espaço não foi pensada apenas como produto de recursos e funções de cada grupo e atividade na competição, mas também estava associada a um ciclo ecológico de invasão-competição-sucessão-acomodação, válido não apenas para atividades e residências, mas também para grupos étnicos (ethnic succession eresidencial invasion). A diferença entre esses processos e as formas naturais de competição ( válidas para plantas e ani- mais) estaria na capacidade humana de transformar as condições ambientais (Bulmer, 1984).

Ao longo das décadas de 1940 e 1950 a ecologia urbana foi revista, reduzindo a ênfase na competição e aumentando a importância da cooperação. Enquanto para a primeira Escola de Chicago, a estruturação do espaço era um produto da luta dos indivíduos e grupos por recursos escassos, para a segunda versão da tradição ecológica, a distribuição socioespacial seria uma adaptação funcional de cada espaço particular a transformações provocadas na sociedade urbana como um todo. A sociedade seria um sistema que, tendendo à homeostase, imprimiria funções diversas a cada uma de suas partes. U ma transformação em determinada configuração espacial representaria uma mudança homeostática das partes daquele sistema.

Para concluir essa rápida discussão a respeito de al gumas concepções de como se estrutura o espaço intra-urbano, deve-se assinalar a contribuição da chamada escola neoclássica (Alonso, 1964). Esse autor construiu uma teoria da renda da terra urbana a partir das simplificações características das análises da economia espacial - a redução de todas as escolhas e estratégias individuais ao cálculo econômico racional, a consideração de todas as empresas como firmas indiferenciadas, a inexistência de regulamentações estatais e a desconsideração de quaisquer características topográficas, considerando o território uma superfície isotrópica. Os preços da terra urbana seriam o resultado de um leilão entre os diversos usos (e grupos diferentes de usuários no interior de cada uso). 0 modelo espacial resultante incorpora diversos fenômenos associados às diferentes curvas de preferências dos agentes econômicos e dos grupos sociais (M arques, 1998). Não há nenhuma forma urbana específica definida a priori, ao contrário dos modelos ecológicos, mas um resultado contingente da definição das preferências. Esse modelo permite sugerir uma explicação para a localização, até então aparentemente contraditória, de pobres e ricos nas cidades americanas: pobres se localizariam nas regiões mais centrais e de maior renda da terra por priorizarem a acessibilidade, mesmo que para isto tivessem que consumir pouca terra (áreas de alta densidade), considerando suas limitações orçamentárias. Camadas ricas da população, ao contrário, não se incomodariam com local izações mais distantes (por utilizarem transporte individual) para poderem consumir maiores quantidades de terra. Obviamente de- 
ve-se contrastar e relativizar essa conclusão, a partir de outras constatações e estudos como os que se encontram em análises das cidades européias e asiáticas (Chamboredon e Lemaire, 1970; Brun e Rhein, 1994; Preteceille, 1997; M arcuse e Kempen, 2000), com especial atenção para aqueles que debatem as conseqüências da globalização econômica nas aglomerações metropolitanas. $\mathrm{H}$ averia grupos sociais de alta renda para quem a localização central está associada a um padrão de vida específico altamente valorado, o que tem levado os centros das chamadas "cidades globais" a um intenso processo de elitização (Sassen, 1998; 2001; Fossaert, 2001).

\section{Resultados: a metodologia}

A metodologia proposta é a mesma já desenvolvida em trabalhos anteriores ( $N$ ajar, 1997; 1998 e 2000), com o seguinte diferencial: a) validá-la através da comparação e discussão com experiências semel hantes; b) aplicá-la para os dados censitários de 1996 e 2000, produzindo análises comparativas; e c) explorar outras dimensões analíticas, conforme detalhado no item O bjetivos. Em seguida, são apresentados, esquematicamente, os principais passos metodológicos:

1) estudo exploratório;

2) seleção/agrupamento de variáveis;

3) construção de indicadores;

4) análise fatorial:

- análise de discriminantes com a finalidade de investigar se residir em setor censitário classificado como do tipo $=0$ (aglomerado normal) ou do tipo $=1$ (aglomerado subnormal) faz diferença do ponto de vista das variáveis escolhidas para análise. Isso é relevante para saber se poderíamos considerar o conjunto dos setores censitários, ou se seria recomendável uma abordagem separada dos setores tipo zero e tipo um. Para os dados do Censo de 1991, o teste de hipótese ( $\mathrm{H}_{0}$ : as médias dos grupos são iguais), nos indicou que $\mathrm{H}_{0} \mathrm{NÃO}$ devia ser rejeitada. O u seja: as médias dos grupos NÃO apresentavam diferenças significativas e conseqüentemente puderam ser examinados em conjunto.

- análise de componentes principais, dividida em três etapas: (1) exame da matriz de correlação de todas as variáveis, para eliminação daquelas que apresentarem coeficiente de correlação muito alto; (2) extração dos fatores; e (3) cálculo dos escores para cada fator escolhido.

- análise de cluster, sendo empregado parageração dos clusters o método de Ward (Aldender- fer, 1984), em que, para cada cluster, as médias para todas as variáveis são calculadas, e, então, para cada caso, o quadrado da distância euclidiana é calculado. Essas distâncias são somadas para todos os casos. A cada etapa, os dois clusters que nascem são aqueles que resultam no menor acréscimo na soma total das distâncias ao quadrado dentro do cluster. Para os dados do Censo 1991, após a análise dos dendrogramas (esquemas classificatórios) o número de clusters foi escolhido como 6 ou 7;

5) definição e análise de categorias sociais (Índice de Qualidade Social - IQS).

Com vista a produzir o que chamamos "mapa social" da RM RJ. Agrupamos os setores censitários anal isados em categorias mais abrangentes, tentando caracterizá-las segundo os três fatores resultantes da análise de componentes principais. Com a classificação feita para cada caso, foram gerados mapas para visualização da distribuição das características dos fatores. A avaliação desses resultados nos sugeriu utilizarmos sete categorias socioeconômicas. A caracterização de cada uma das sete categorias foi obtida a partir do ranking dos setores no interior de cada categoria;

6) Geração de mapas.

Os resultados já comentados das análises apresentadas em N ajar (1997, 1998 e 2000) levaram a propor o índice de qualidade social (IQS) como índice sintetizador da metodologia. Ele é de fato uma escala de classificação dos setores censitários RM RJ, resultante do exame das variáveis envolvidas com cada fator da análise de componentes principais e dos procedimentos analíticos apresentados. Desta forma, o resultado final nos possibilitou classificar as áreas da RM RJ da seguinte forma:

Grupo I (IQS muito alto): setores censitários de mais alto status social. Estes são caracterizados pelos maiores rendimentos, maior proporção de chefes de domicílios com curso superior, mestrado ou doutorado; baixa densidade domiciliar, indicando famílias pequenas, domicílios grandes e em sua maioria próprios; condições de saneamento excelentes - coberturas em torno de $100 \%$; e concentração de população com mais de 65 anos de idade superior à média do município.

Grupo II (IQS alto): em termos de renda do chefe do domicílio, este grupo apresenta, em geral, a metade do anterior. No que se refere a anos de estudo do chefe, a distribuição fica em torno de $29 \%$ para o segundo grau e $38 \%$ para a categoria superior ou mais; a densidade domiciliar também é baixa, e em sua maioria os 
domicílios são próprios. As condições de saneamento continuam muito boas, da mesma forma que o grupo anterior, e a concentração de população na terceira idade é, geralmente, a maior de todos os grupos.

Grupo III (IQS médio): a distribuição dos anos de estudo do chefe do domicílio indica primeiro e segundo graus completos, e a renda cai mais que a metade com relação ao grupo II. A densidade de pessoas no domicílio aumenta consideravelmente, indo para 2,87 pessoas em média, distribuindo-se em 1,56 dormitórios por domicílio, enquanto nos grupos anteriores essa relação era de 2,96 por 1,97 (grupo II); e 3,20 por 2,14 (grupo I). A maior parte dos domicílios é alugada. Com relação às condições de saneamento, continuam excelentes. A proporção de população com 65 anos de idade ou mais fica em torno de $10 \%$. No que se refere às mulheres chefes de domicílio, é maior a proporção nesse grupo, indicando, possivelmente, famílias menores, maior número de mulheres separadas e maior participação de mulheres no mercado de trabalho.

Grupo IV (IQS médio/baixo): é o maior grupo. Em termos de rendimento é apenas um pouco mais baixo que o anterior. Há um aumento na proporção de chefes de domicílio com quatro a sete anos de estudo (32,7\%) , com relação ao grupo anterior, sugerindo aumento na proporção de mão-de-obra desqualificada. As faixas de 8 a 10 e de 11 a 14 anos de estudo continuam iguais. As famílias tendem a ser maiores, e os domicílios menores. A maior parte deles é própria (58\%), as condições de saneamento são boas, caindo a proporção de pessoas com 65 anos de idade ou mais.

Grupo V (IQS baixo): a proporção do número de anos de estudo do chefe do domicílio distribui-se entre um a sete anos, ou seja, em geral a metade dos chefes de domicílio possuem baixo grau de escolaridade (alfabetização/elementar); com relação aos rendimentos, esse grupo situa-se abaixo da média do restante do próprio município, como os grupos II eIV, e aproximadamente 7,5 vezes abaixo do grupo I. A maior parte dos domicílios é própria $(84,96 \%)$, havendo um aumento considerável dos domicílios com mais de cinco moradores. A média de pessoas por banheiro chega, nesse grupo, a 3,84. Juntamente com o grupo I, éonde encontramos a maior proporção de mulheres em idade fértil $(55,7 \%)$ - o grupo I está em torno de $56 \%$. No que se refere ao abastecimento de água e esgotamento sanitário, a situação é bastante satis- fatória, com os patamares se mantendo elevados. Com relação ao lixo, o patamar fica abaixo dos $90 \%(82,08 \%)$, com um significativo aumento da coleta indireta.

Grupo VI (IQS baixo/muito baixo): da mesma forma que no grupo anterior, a escolaridade do chefe do domicílio é baixa, estando compreendida entre a alfabetização e o elementar, mas com uma proporção maior (60\%). Os rendimentos são baixos - aproximadamente 12 vezes abaixo do grupo I - , a proporção da população analfabeta sobe dramaticamente para al go em torno de $30 \%$; mais ou menos $86 \%$ dos domicílios são próprios, mas pequenos, sendo a taxa de utilização do domicílio como dormitório (dormitório por cômodo) em torno de 0,4 ; 3,87 pessoas por banheiro e quase três pessoas por dormitório. Pela primeira vez, a proporção de domicílios com água não acompanha de perto a existência de instalação sanitária. A proporção de domicílios que não possuem instalação sanitária cresce muito, saindo da casa dos decimais para chegar a $6,5 \%$. Os problemas de lixo são dramáticos, e a proporção de população na terceira idade está em torno de 3\%.

Grupo VII (IQS muito baixo): neste grupo, encontram-se as piores condições sociais. A renda é 14 vezes menor que a do grupo I; a proporção da população analfabeta, em torno de $37 \%$, e a maioria dos chefes do domicílio (aproximadamente $62 \%$ ) é apenas alfabetizada ou possui apenas o curso elementar, representando certamente a mão-de-obra mais desqualificada, juntamente com o grupo VI. Com mais de três pessoas por domicílio e quatro por banheiro, esse grupo apresenta carências básicas de saneamento e situação alarmante de coleta de lixo.

\section{Comentários finais}

A coleta de dados sobre a distribuição espacial de propriedades significativas da superfície terrestre há muito tempo tem sido uma importante atividade das sociedades organizadas. D esde as civilizações primitivas até os tempos modernos, mapas têm sido utilizados para retratar dados espaciais. Os mapas tiveram uso intensivo no Império Romano, declinando juntamente com o declínio deste. Somente no século 18, seu uso voltou a crescer na Europa, a partir da necessidade dos governantes da época de real izar um mapeamento sistemático de suas terras (Burrough, 1990; Aronoff, 1989). Remontam desse período os primeiros mapeamentos de doenças. 
Em 1768, o médico escocês James Lind publicou um livro intitulado: An essay on diseases incidental to Europeans in hot climates: with the method of preventing their fatal consequences (Barret, 1991). No século 19, o clássico estudo de John Snow (1990) utilizou técnicas de mapeamento para analisar a epidemia de cólera ocorrida em 1854 na região de Soho, Londres. Ele demonstrou uma associação espacial entre mortes por cólera e suprimento de água por diferentes bombas públicas de abastecimento, identificando assim a origem da epidemia, mesmo sem conhecer seu agente etiológico. 0 trabalho deJohn Snow é uma referência até os dias atuais.

Entretanto, só recentemente começou-se a utilizar de forma sistemática as técnicas de mapeamento para a análise da distribuição espacial de eventos de saúde. Esse crescimento devese em muito ao avanço dos recursos computacionais, e em especial das técnicas de geoprocessamento que, entre outras tarefas, permitem a rápida atualização de mapas, sem a necessidade de refazêlos manualmente. Além disso, a incorporação das técnicas de análise estatística espacial ao geoprocessamento pode aprimorar 0 entendimento do espaço na produção e difusão de doenças e outros agravos à saúde, tornandose assim um instrumento valioso não apenas de visualização de dados georreferenciados, mas também de modelagem espacial.

Por sua vez as ferramentas de análises espaciais desenvolvidas nos últimos anos podem potencializar a identificação de padrões espaciais de morbidade ou mortalidade e os fatores associados a esses padrões. Segundo Gatrell \& Bailey (1996), os métodos para análise espacial podem ser divididos em: visualização, análise exploratória de dados e modelagem espacial. Este último método é utilizado quando se pretende testar formalmente uma hipótese ou estimar relações. D evido ao fato de a ocorrência de um específico evento de saúde em uma região estar provavelmente correlacionada à sua ocorrência em regiões vizinhas, não se pode necessariamente utilizar modelos estatísticos clássicos, que assumem independência entre os eventos.

Nesse sentido, pode-se distinguir claramente que os procedimentos utilizados para executar a análise espacial não se resumem simplesmente ao mapeamento dos eventos, exigindo também a necessidade de se adicionar um novo significado aos dados. Diversos procedimentos vêm sendo utilizados para a análise espacial, notadamente, as técnicas de geoprocessamento, de análise geoestatística, de dados em treliça - lattice e de ponto-evento - point patterns (Cliff \& Ord, 1981; Cressie, 1993; Deutsch \& Journel, 1998; Kaluzny et al., 1996; M edronho, 1995; 1999).

Entretanto, a despeito do desenvolvimento das técnicas quantitativas para o estudo do tempo (séries temporais), o estudo de problemas relacionados ao espaço não acompanhou este desenvolvimento. Com o avanço recente das técnicas de análise espacial (geoprocessamento, geoestatística, etc.) aplicadas a outros campos do conhecimento, pode-se perceber a grande contribuição que as mesmas estão trazendo para a saúde coletiva. Entretanto, essa incorporação tem se dado de forma ainda pontual e esparsa, não contemplando os múltiplos aspectos da análise espacial (Campos et al., 2002). Por isso, faz-se necessário integrar de forma sistemática todos esses aspectos no sentido de aprimorar-se o entendimento da dependência espacial nos estudos em Saúde.

A análise conjunta de componentes de variação, determinístico eestocástico, como éo caso de model os espaciais, carece de sistematização, não se constituindo atualmente em procedimento rotineiro dos programas de computação. Da mesma forma a incorporação da estrutura de depen dência espacial nos estudos ecológicos está sendo objeto de estudo e operacionalização (Szwarcwald \& Leal, 1997). Ao lado disto, o desenvolvimento e/ou adaptação de mode los aptos a lidar com uma estrutura de dependência no espaço e no tempo permanece ainda como desafio, cujas dificuldades de ordem teórica e prática têm restringido sua aplicação.

Acredita-se, assim, que o uso ampliado das técnicas de análise espacial disponíveis, junto à elaboração de novos procedimentos que visem solucionar os obstáculos metodológicos encontrados na prática, certamente virá aumentar 0 rol das aplicações da análise estatística espacial aos estudos de ocorrências de eventos na área da Saúde.

Lembrando que a desigualdade espacial, sendo um aspecto da desigualdade em geral, tem sua importância reforçada quando segregações espaciais e regionais alinham-se com tensões políticas e étnicas, e que o crescimento da desigualdade espacial está relacionado também a uma maior abertura das economias e à adaptação ao processo de globalização, ten do por conseqüência não só uma submissão à dinâmica global, mas também uma imensa diferenciação no interior das grandes metrópoles, espera-se ter apresentado, o que pretende ser 0 arcabouço básico de uma colaboração conjunta no desenvolvimento de tipologias de análise socioespacial. 


\section{Referências bibliográficas}

Abreu MDA 1987. A evolução urbana do Rio de Janeiro. Zahar Ed.-I planRio, Rio de Janeiro.

Aldenderfer M S \& Blashfield RK 1984. Cluster analysis. Sage Publications, N ewbury Park, CA.

Alonso W 1964. Location and land use. Harvard University Press.

Aronoff S 1989. Geographic information systems: a management perspective. WDL Publications, Canada.

Barret FA 1991. 'Scurvy' Lind's M edical Geography. Social Science and M edicine 33:347-53.

Brun J \& Rhein C 1994. La ségrégation dans la ville: concepts et mesures. Editions L ' $\mathrm{H}$ armattan, Paris.

Bulmer M 1984. The Chicago School of Sociology: institutionalization, diversity and the rise of sociological research. The University of Chicago Press, Chicago.

Burrough PA 1990. Principles of geographical information systems for land resources assessment. Oxford U niversity Press, Nova York.

Campos M R, Valencia LIO, Fortes BPM D, Braga RCC \& M edronho RA 2002. Distribuição espacial da infecção por Ascaris Lumbricóides. Revista de Saúde Pública 36(1):69-74.

Chamboredon J-C \& Lemaire M 1970. Proximité sociale et distance spatiale: les grandes ensembles et leur peuplement. Revue Française de SociologieXI (1):3-33.

Cliff AD \& Ord JK 1981. Spatial processes: models and applications. Pion, Londres.

Cressie N 1993. Statistics for spatial data. Wiley \& Sons, Nova York.

Deutsch CV \& Journel AG 1998. GSLIB: Geostatistical Software Library and U ser's Guide. (2a ed.). Oxford University Press, N ova York.

Fossaert R 2001. Les villes mondiales, villes du système mondial. H érodote: revue de géographie et de géopolitique (101):11-24.

Gatrell AC \& Bailey TC 1996. Interactive spatial data analysis in medical geography. Social Science and M edicine 42(6):843-855

Kaluzny SP, Vega SC, Cardoso TP \& Selly AA 1996. S+SpatialStats: user's manual for Windows and U nix. M athSoft, Inc., Seattle.

M achado da Silva LA 1967. A política na favela. Cadernos Brasileiros 9(3):35-47.

M arcuse P \& Kempen RV 2000. Globalizing cities: a new spatial order? Blackwell Publishers Ltd., Bodmin, Cornwall.

$M$ arques E 1993. D esigualdades sociais e infra-estrutura urbana: a produção do saneamento no Rio de Janeiro. Dissertação de mestrado. Instituto de Pesquisas e Planejamento U rbano e Regional/UFRJ, Rio de Janeiro.

$M$ arques $E$ 1998. Os modelos espaciais como instrumento para o estudo dos fenômenos urbanos. In A Najar $\& E M$ arques (orgs.). Saúde e espaço: estudos metodológi cos etécnicas de análise. Fiocruz, Rio de Janeiro.

M edronho RA 1995. Geoprocessamento e saúde: uma nova abordagem do espaço no processo saúde-doença. N ect/ Fiocruz, Rio de Janeiro.

M edronho RA 1999. Avaliação do método geoestatístico no estudo da distribuição espacial da hepatite $A$. Tese de doutorado. Escola Nacional de Saúde Pública/Fiocruz, Rio de Janeiro.

Najar AL 1997. Desigualdades sociais e análise espacial: uma aplicação para o município do Rio de Janeiro uti- lizando sistemas de informações geográficas. Tese de doutorado. IUPERJ, Rio de Janeiro.

Najar AL 1998. Desigualdades de bem-estar social no município do Rio de Janeiro: um exemplo de aplicação da ferramenta SIG. In AL Najar \& E M arques 1998. Saúde e espaço: estudos metodológicos e técnicas de análise. Fiocruz, Rio de Janeiro.

$\mathrm{N}$ ajar AL et al. 2000. Rio 40 graus: metodologia de seleção de áreas para minoração de desigualdades de bem-estar e promoção da saúde na região metropolitana do Rio de Janeiro ( 3 volumes). ENSP/Fiocruz, Rio de Janeiro.

$\mathrm{N}$ ajar AL et al. 2002. Desigualdades sociais no município do Rio de Janeiro: uma comparação entre os censos 1991 e 1996. Cadernos de Saúde Pública 18(supl.):89-102.

Oliveira R 1988. Sobre o pensamento antropológico. Tempo Brasileiro. Rio de Janeiro.

Park R, Burguess E \& M ackenzie R 1925. The city. The University of Chicago Press, Chicago.

Pinçon-Charlot M , Preteceille E \& Rendu P 1986. Ségré gation urbaine: classes sociales et equipements collectifs en région parisienne. Ed. Anthropos, Paris.

Preteceille $E \&$ Valadares $L$ 2000. A desigualdade entre os pobres - favela, favelas, pp. 459-485. In Ricardo Henriques (org.). D esigualdade e pobreza no Brasil. I pea, Rio de Janeiro.

Preteceille E 1997. Ségrégation, classes et politique dans la grande ville, pp. 99-127. In A Bagnasco \& PL Galès. Villes en Europe. Editions La Découverte, Paris.

Sassen S 1991. The global city: N ew York, London, Tokyo. Princeton University Press, N ova Jersey.

Sassen S 1998. Globalization and its discontents: essays on the new mobility of people and money. The N ew York Press, Nova York.

Sassen S 2001. The global city: N ew York, London, Tokyo. Princeton University Press, N ova Jersey.

Snow J 1990. Sobre a maneira de transmissão do cólera. Hucitec, São Paulo.

Szwarcwald CL \& Leal MC 1997. Estatística espacial na análise exploratória de dados epidemiológicos. Saúde e movimento. Fiocruz, Rio de Janeiro.

Timms D 1971. The urban mosaic: towards a theory of residential differentiation. Cambridge University Press, Cambridge, EUA.

Valladares LP 2001. Favela.com. Paper apresentado no Forum America Latina H abitar 2000, Salvador, Bahia, 15 a 18 de maio 2001.

Velho $O$ 1973. 0 fenômeno urbano. Zahar Ed., Rio de Janeiro.

Ventura Z 1994. Cidade partida. Companhia das Letras, São Paulo.

Vetter D 1981. A segregação residencial da população economicamente ativa na região metropolitana do Rio de Janeiro, segundo grupos de rendimento mensal. Revista Brasileira de Geografia 43(4):587-603.

Vetter D 1982. Quem se apropria dos benefícios líquidos dos investimentos do Estado em infra-estrutura urbana? U ma teoria de causação circular. In L M achado da Silva (org.). Solo urbano: tópicos sobre o uso da terra. Série D ebates urbanos n. 1. Zahar Ed., Rio de Janeiro.

Artigo apresentado em 20/10/2002

Aprovado em 11/11/2002

Versão final apresentada em 5/12/2002 